\title{
The role of segmentation in prospective and retrospective time estimation processes
}

\author{
DAN ZAKAY, YEHOSHUA TSAL, MASHA MOSES, and ITZHAK SHAHAR \\ Tel Aviv University, Tel Aviv, Israel
}

\begin{abstract}
In five experiments, we investigated the effects of the segmentation level of an interval on its perceived duration. A prospective paradigm and an absolute time estimation method were used in two experiments, and in two others we used a retrospective paradigm and a comparative estimation method. A positive relationship was obtained between segmentation level of the estimated interval and its perceived duration under retrospective-comparative conditions for both auditory and tactual stimuli, but no relationship was found under prospective-absolute conditions. The paradigm, estimation method, and segmentation level were jointly manipulated in the fifth experiment. The impact of segmentation was significant under retrospective (both absolute and comparative) and close to significant under prospective-comparative conditions. These findings suggest that high-priority events are perceived and coded as contextual changes and that the impact of segmentation on time estimation is mediated by memory processes.
\end{abstract}

An important distinction in time estimation research is that between prospective and retrospective methods. In prospective time estimation, subjects are informed that they will later be asked to estimate the duration of a given interval, whereas in retrospective time estimation this information is not known in advance. The two methods have produced different patterns of results, suggesting that time estimation under these two conditions is mediated by different processes. Accordingly, Block (1974) termed prospective and retrospective time estimates experienced and remembered durations, respectively.

Zakay $(1989,1990)$ pointed out that in prospective conditions, but not in retrospective conditions, attention is directed in real time to information that is related to the passage of time. Retrospective estimates are based on memory-related processes, because duration estimates necessitate the retrieval of time-relevant information. Prospective estimates, on the other hand, are mediated by processes of real-time temporal information such as grouping or counting of temporal elements (Jones \& Boltz, 1989). This proposed distinction between the two types of processes is supported by a wide variety of studies that have shown different patterns of results for prospective and retrospective time estimates (e.g., Block, 1992; Hicks, Miller, Gaes, \& Bierman, 1977; Hicks, Miller, \& Kinsbourne, 1976; McClain, 1983; Thomas \& Brown, 1974; Zakay, 1989, 1992a, 1992b; Zakay, Nitzan, \& Glicksohn, 1983). Zakay (1989) further argued that underprospective conditions, a negatively monotonic relationship is typically obtained between magnitude of duration

Address correspondence to D. Zakay, Department of Psychology, Tel Aviv University, Ramat Aviv, Tel Aviv 69978, Israel.

-Accepted by previous editor, Margaret Jean Intons-Peterson estimates and nontemporal information processing load (e.g., Grondin \& Macar, 1992; Zakay, 1992b), whereas the respective relationship under retrospective conditions is positively monotonic (e.g., Ornstein, 1969; Zakay \& Fallach, 1984). This negative relation is best accounted for by capacity models of time estimation (e.g., Hicks et al., 1977; Thomas \& Weaver, 1975; Zakay, 1989), which assume that duration estimates are a function of the amount of temporal information processed by a cognitive timer (Berlyne, 1966) whose operation requires cognitive resources that are shared with concurrent nontemporal information processes. Thus, a high level of nontemporal load reduces the number of temporal units processed by the cognitive timer, consequently producing shorter estimated durations.

The positive relationship between estimated durations and load obtained under retrospective conditions is best explained by memory-based models, which postulate that certain types of information stored in memory during the relevant interval provide the basis for time estimation. Block $(1978,1989)$ proposed that duration estimates are correlated with the amount of changes in cognitive context during the estimated interval. For instance, Block and Reed (1978) suggested that varying the level of processing might influence retrospective judgments, presumably because processing at different levels produces more contextual changes than processing at a single level. Thus, the higher the level of nontemporal processing during the interval, the greater the number of contextual changes taking place in that interval and, most probably, the number of contextual changes coded and stored in memory (e.g., Poynter, 1989), leading to higher time estimates.

Clausen (1950) distinguished between comparative and absolute time estimation methods. Zakay (1990) proposed that estimation method is also an important factor in determining the involvement of either memory or attentional 
processes in time estimation. In comparative time estimation, the duration of the target interval is estimated by comparing it with that of a standard interval either preceding or following it. In absolute time estimation, the duration of the interval is estimated without any reference to another external interval. Thus, the comparative time estimate relies heavily on memory processes because the representation of both the target and the standard intervals must be retrieved from memory. In the absolute estimate, on the other hand, the reliance on memory processes is reduced because the duration of the target interval can be based on information retrieved directly from the cognitive timer (Berlyne, 1966), with no need for memory search. Thus, it is proposed in the present paper that time estimation under a retrospective paradigm combined with comparative time estimation methods (RC) is dependent on memory processes to a higher degree than is time estimation under a prospective paradigm combined with absolute time estimation methods (PA). When either a prospective paradigm is combined with a comparative time estimation method (PC), or a retrospective paradigm is combined with an absolute estimation method (RA), the contribution of memory processes to time estimation is expected to be lower than that in the RC condition but higher than that in the PA condition. In the RA and PC conditions, time estimation is a function of both the input retrieved from the cognitive timer and the amount of contextual changes retrieved from memory storage (Thomas \& Weaver, 1975; Zakay, 1989). Therefore, the influence of contextual changes is expected to be partial in the RA and PC conditions.

Poynter (1983, 1989; Poynter \& Homa, 1983) presented a segmentation model, suggesting that events filling a to-be-estimated interval may serve as significant segmentation markers. Processing these events can generate temporal referents in memory for subsequent reconstruction of the interval duration. Poynter (1983) manipulated segmentation level by either clustering (low segmentation) or distributing (high segmentation) three high-priority events (HPEs) to which subjects were instructed to pay special attention during the presentation of a word list. Indeed, the interval was retrospectively perceived as longer for the high-segmentation than for the low-segmentation condition. There were, however, no differences in recall or recognition between the two conditions, implying that perceived duration is not determined by the amount of information retrieved from memory, but rather by "the degree to which memorial representations of processed events segment experience"' (Poynter, 1983, p. 81).

It is our contention that HPEs provide contextual changes that are coded and stored in memory, and hence segmentation is a particular case reflecting the general effect of contextual changes. Thus, the impact of segmentation on time estimates depends on the relative role of memory processes in producing time estimates. Therefore, segmentation level should not affect time estimation that is based on real-time temporal information processing (e.g., PA estimates) and should have an intermediate effect when the role of memory processes is partial (e.g., RA and PC estimates).

The purpose of the present study was to empirically test these notions by comparing time estimates under different combinations of paradigm and time estimation methods. It should be noted that although the level of segmentation has indeed previously been found to be positively correlated with duration estimates under RC conditions (Poynter, 1983; Poynter \& Homa, 1983; Zakay \& Feldman, 1993), the influence of segmentation on time estimation under RA, PA, and PC conditions has not been tested before. Another purpose of this study was to examine the generality of the segmentation effects on time estimation in terms of stimulus type and perceptual modality. In previous studies, level of segmentation was manipulated only with verbal stimuli. In the present study, tactual stimuli were presented as well, in order to investigate whether these effects can be extended beyond a single modality. If evidence that the influence of segmentation is not modality dependent is provided, then this will support the notion that segmentation is determined by any stimuli creating contextual changes.

\section{Overview of Experiments}

Five experiments were conducted. The first two experiments were an investigation of the effect of segmentation level on time estimates for verbal stimuli presented auditorily under the RC condition (Experiment 1) and PA condition (Experiment 2). In Experiments 3 and 4, we examined the effect of segmentation level on time estimates for tactual stimuli under the RC condition (Experiment 3) and PA condition (Experiment 4). In the RC condition, comparative length estimates were used analogically to represent time. In the PA condition, absolute estimates were reproduced and measured in seconds. The two treatment combinations were selected so that the first one maximized the involvement of memory-based processes (RC), whereas the latter maximized attentional processes directed to temporal information processing (PA). The RC and PA conditions were treated in separate experiments, because they involve different methods of time estimation. In the fifth experiment, the two variables were jointly manipulated in order to assess the effects of each combination (RC, RA, PC, and PA).

\section{EXPERIMENT 1}

The first experiment was nearly an exact replication of Poynter's (1983) study. Its purpose was to show that, under retrospective-comparative (RC) conditions, increasing the segmentation level would produce longer estimated durations.

\footnotetext{
Method

Subjects. Thirty-eight subjects fulfilling a course requirement were randomly assigned to two equal groups.
} 
Stimuli. Two tapes were prepared, each including two recordings. The first recording contained a 30 -sec empty interval, beginning with the word "start" and ending with the word "end." The second recording lasted for $155 \mathrm{sec}$ and included 30 words presented with a uniform voice and had equal intensity. Twenty-seven words were nouns of equal frequency in Hebrew, had equal associative value, and were emotionally neutral (as determined in a pilot study). Three words were the last names of famous Israeli politicians. According to Poynter's (1983) rationale, politicians' names embedded among nouns become HPEs because of their distinct meaning, and their arrangement within the list can thus affect the level of segmentation. In the low-segmentation condition, the politicians' names were placed in Positions 1,2, and 3. In the highsegmentation condition they were placed in Positions 10, 20, and 27 . The positions of the remaining nouns in the list were determined randomly. The duration of each word was approximately $1 \mathrm{sec}$, and the empty interval between adjacent words was $4 \mathrm{sec}$. The recording began with the word "start" and ended with the word "end."

The perceived interval duration was measured by a comparative estimation method. The subjects were presented with a page showing two parallel horizontal lines, $50 \mathrm{~mm}$ apart. Both lines originated from a common left-hand margin, but the bottom line was extended farther to the right. The top line subtended $30 \mathrm{~mm}$ and represented the empty recording. The bottom line subtended $300 \mathrm{~mm}$, and the subjects were instructed to divide it into two segments so that the left segment represented the estimated duration of the recorded word list relative to the empty recording represented by the top line.

As used by Block (1974), the following memory tests were also conducted in order to examine the relationship among segmentation level, the number of words remembered, and time estimates.

1. Recalled number of events (RNE). The subjects were asked to report the number of words presented in the list. The purpose of this measure was to examine the relation between the remembered number of stimuli and the estimated interval, independently of the ability to retrieve the words from memory.

2. Free recall $(F R)$. The subjects wrote down the number of words they could remember from the list. The number of words recalled correctly served as a measure of FR.

3. Recognition measure (CG). The subjects were presented with a recorded list of 50 nouns and 10 names of politicians that were randomly intermixed. Half of the words appeared in the original experimental list. They were instructed to indicate which of the words they had heard before. The number of words correctly recognized was corrected for guessing by subtracting the number of false alarms from the total number of recognized words (Baddeley, 1990). This corrected number served as a measure of CG.

Design. The thirty-eight subjects were randomly assigned to two equal groups, the high-segmentation and low-segmentation conditions. Within each group, 10 subjects were randomly selected for the free-recall test and the remaining subjects were assigned to perform the recognition test.

Procedure. Each subject was tested individually in a soundproof room; they were told that the purpose of the experiment was to investigate memory. First, the subject was instructed to put on headphones and was presented with a short trial recording in order to provide experience with them. Then the subject was instructed to pay attention to the forthcoming interval, beginning with the word "start" and ending with the word "end." This was followed by the empty recording. Then the following instructions were given: "You will now hear a recorded list of words beginning with the word 'start' and ending with the word 'end.' Listen to the tape carefully and try to remember as many words as you can, since you will later be presented with a memory test. Pay special attention to last names of politicians that may be embedded in the list. Wait for further instructions at the end of the list presentation." The recorded word list followed the instructions. Following the presen- tation, the subject estimated the duration of the recorded list, as specified above, and was tested for the RNE followed by either the FR or CG test.

\section{Results and Discussion}

There were no differences in time estimates between the subjects who performed the FR test and those performing the CG test. The mean duration estimates were $132.21 \mathrm{~mm}(S D=57.03)$ and $218.37 \mathrm{~mm}(S D=58.34)$ for the low- and high-segmentation groups, respectively. This difference was significant $[t(36)=4.41, p<.01]$. None of the memory measures indicated any significant difference between the low- and high-segmentation groups. The mean RNE, FR, and CG measures were 27.89, 13.25 , and 20.95 , respectively, for the low-segmentation group, and $31.21,14.50$, and 21.05 , respectively, for the high-segmentation group. HPEs were perfectly recalled and recognized. A stepwise multiple regression analysis, including segmentation level and all memory measures as independent variables, was performed in order to explore the relative impact of each measure on time estimates. A multiple correlation of .54 with magnitude of time estimates was obtained. The only independent variable that significantly contributed to the regression equation was segmentation level $[F(1,36)=15.01, p<.01]$.

The present findings replicate those of Poynter (1983) in showing that, under the $\mathrm{RC}$ condition, the higher the segmentation level, the longer the perceived time interval. The analysis of the memory measures indicates that the effect of segmentation is not explained by an increase in memory accuracy as reflected by FR or CG, thus supporting the notion that HPEs do not facilitate the process of recall or recognition and that $\mathrm{RC}$ time estimates are primarily related to contextual changes that are caused by HPEs. The present results support the notion that time estimates obtained in the RC condition are largely mediated by memory-based processes.

\section{EXPERIMENT 2}

In the second experiment, prospective time estimates were measured in the absolute method. The purpose was to show that under these conditions, increasing the segmentation level would not influence the estimated durations because this paradigm minimizes the involvement of memory processes.

\section{Method}

Subjects. Fifty-two subjects fulfilling a course requirement were randomly assigned to two equal groups, representing the highsegmentation and low-segmentation conditions. Within each group, half of the subjects were tested for recall and the other half were tested for recognition.

Stimuli and Procedure. The subjects were given the same instructions as used in Experiment 1, but were also informed before the presentation of the word list that they would later be asked to estimate its duration. The perceived duration of the recorded word list was reproduced by pressing a button to indicate the beginning and the end of its perceived duration without any reference to the empty interval. The buttonpress activated a hidden electronic timer 
that measured the duration to the nearest $100 \mathrm{msec}$. In all other respects, Experiment 2 was identical to Experiment 1.

\section{Results and Discussion}

There were no differences in time estimates between the subjects tested for recall and those tested for recognition. The mean estimates were $96.22 \mathrm{sec}(S D=32.18)$, and $94.89 \mathrm{sec}(S D=42.02)$ for the low- and highsegmentation groups, respectively. This difference was not significant $[t(50)=.13, p>.50]$. The average measures of RNE, FR, and CG were $32.76,12.69$, and 20.02 for the low-segmentation group, respectively, and 30.73 , 12.19 , and 20.34 for the high-segmentation group, respectively. None of these memory measures produced any reliable difference between the two groups $(p>.50$ for each measure). HPEs were perfectly recalled and recognized in both conditions. A multiple regression analysis, similar to that performed in Experiment 1, revealed no significant correlation $(R=.11)$, and none of the independent variables were found to contribute significantly to the regression equation.

The present findings show that segmentation level has no influence on time estimates under PA conditions and that this is not caused by any recall or recognition deficit for either ordinary words or HPEs. Hence, segmentation and, most probably, contextual changes do not play a role in PA time estimates, thus supporting the notion that time estimation under these conditions is not mediated by retrieval of content information from memory.

\section{EXPERIMENTS 3 AND 4}

\section{General Method}

The purpose of Experiments 3 and 4 was to extend the generality of the differential segmentation effect beyond the auditory modality. Subjects were presented with a tactual recognition task, and HPEs were represented by salient tactual items that were perceptually distinguishable from the remaining items. In Experiment 3 we used the RC condition, and in Experiment 4 we used the PA condition. A successful replication of the results of the first two experiments in the present context would support the notion that processes of time estimation do not depend on the specific modality in which stimuli are perceived during the estimated interval. Moreover, such results would also support the notion that segmentation is determined by any stimuli causing contextual changes.

Tactual task stimuli. The stimuli were $9 \times 9 \mathrm{~cm}$ square plastic surfaces, with a central indented surface depicting a Hebrew letter. The surface of the letter was rough, but the remaining surface was smooth. The height of the indented letter was $0.5 \mathrm{~cm}$. The 11 letters, randomly selected from the Hebrew alphabet, are presented in Figure 1. Three additional stimuli of a different nature were used

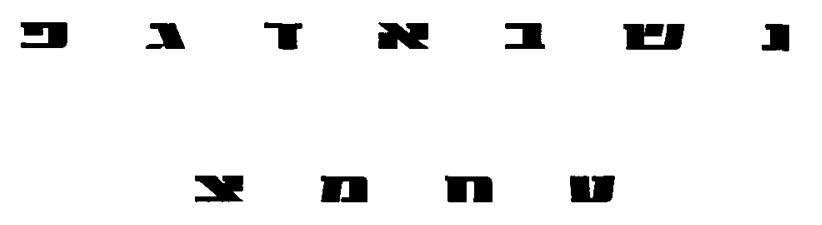

Figure 1. Hebrew letters used as tactual stimuli. as HPEs. These were half of a Ping-Pong ball, a coin, and a piece of fabric. The stimuli were presented inside a $30 \times 30 \mathrm{~cm}$ box. The box had two openings-the front one for the subjects to insert their hands, and the back one for a stimulus-changing device. The task was to identify each stimulus on the basis of tactual sensations. In the low-segmentation conditions the three HPEs were presented as the first three stimuli, whereas in the high-segmentation condition they were presented in Positions 3, 8, and 12.

\section{EXPERIMENT 3}

\section{Method}

Subjects. Thirty subjects fulfilling a course requirement were randomly assigned to two equal groups of either high or low segmentation.

Procedure. Each subject was tested individually in a soundproof room. The subjects were instructed that the purpose of the experiment was to test their tactual recognition ability. They were further told that most of the stimuli were Hebrew letters, but that they were to pay special attention to the nonletter stimuli. Each stimulus was presented for $8 \mathrm{sec}$, with an interstimulus interval of $2 \mathrm{sec}$.

The subject inserted the dominant hand into the box upon hearing the instruction "start," and $2 \mathrm{sec}$ later the first stimulus was presented. Upon hearing the word "end," the hand was removed immediately. The total duration of the task was $140 \mathrm{sec}$. The various time intervals were controlled by an electronic timer that was accurate to the nearest $.10 \mathrm{sec}$. The sequence of events was as follows: After ensuring that the instructions were fully understood, a subject placed the dominant hand inside the box for the supposed purpose of getting used to the box. This interval lasted for $35 \mathrm{sec}$; its actual purpose was to serve as a standard for the subsequent time comparison. Following this interval, the tactual task was carried out, and the comparative time estimation and memory tests were conducted. The time estimation procedure was identical to that performed in Experiment 1, with one exception-the lengths of the lines representing the standard and target intervals were $35 \mathrm{~mm}$ and $350 \mathrm{~mm}$, respectively. The memory tests consisted of the RNE and free-recall tests, in which the subjects wrote down a description of each stimulus they could remember.

\section{Results and Discussion}

The mean duration estimates were $140 \mathrm{~mm}(S D=$ $73.91)$, and $218.50 \mathrm{~mm}(S D=78.34)$ for the low- and high-segmentation conditions, respectively. This difference was significant $[t(28)=2.73, p<.01]$. The mean numbers of RNE, letters, and HPEs correctly recalled were $9.06(S D=2.80), 7.97(S D=2.09)$, and 2.70 $(S D=0.46)$, respectively, for the low-segmentation group, and $9.50(S D=2.23), 8.43(S D=2.35)$, and $2.80(S D=$ 0.40 ), respectively, for the high-segmentation group. None of the memory differences between the high- and low-segmentation groups approached statistical significance. A stepwise multiple regression analysis, including segmentation level, with all memory measures as independent variables, showed a multiple correlation of .46 with magnitude of time estimation. The only independent variable that significantly contributed to the regression equation was segmentation level $[F(1,28)=15.93, p<.01]$.

The present results resemble those of Experiment 1, thus supporting the generality of the relationship between segmentation level and time estimates under RC conditions, and strengthening the notion that time estimates under these conditions are indeed mediated by memory- 
based processes, although not by memory for stimulus events per se. The nature of the relevant memory-based processes will be addressed in the General Discussion.

\section{EXPERIMENT 4}

\section{Method}

Subjects. Thirty subjects fulfilling a course requirement were randomly assigned in equal numbers to the high- and lowsegmentation conditions.

Stimuli and Procedure. The stimuli and procedure were identical to those of Experiment 3, except that duration was estimated with the absolute reproduction method used in Experiment 2, and that the subjects were informed prior to the beginning of the tactual task that they would be asked to estimate its duration.

\section{Results and Discussion}

The mean duration estimates were $68.06 \mathrm{sec}(S D=$ $31.44)$ and $72.26 \mathrm{sec}(S D=39.07)$ for the low- and highsegmentation groups, respectively. This difference was not significant $[t(28)=0.31, p>.5]$. The mean number of RNE, letters, and HPEs correctly recalled were $9.56(S D=1.71), 8.53(S D=2.02)$, and $2.66(S D=$ 0.47 ), respectively, for the low-segmentation group, and $9.96(S D=2.31), 8.00(S D=2.84)$, and $2.73(S D=$ 0.44 ), respectively, for the high-segmentation group. None of the memory differences between the high- and low-segmentation groups approached statistical significance. A stepwise multiple regression analysis that included the same variables used in Experiment 3 showed a multiple correlation of .24 with magnitude of time estimation. None of the independent variables contributed significantly to the regression equation.

These results resemble those of Experiment 2, extending its generality beyond the auditory modality, and further substantiating the notion regarding the lack of influence of segmentation level on time estimation under PA conditions.

\section{SUMMARY OF EXPERIMENTS 1-4}

A summary of the findings obtained in the first four experiments is presented in Table 1. A parameter of magnitude of experimental effects was computed for each condition by employing Rosenthal's (1984) formula $\left[R^{2}=\right.$ $\left.\sqrt{t^{2} /\left(t^{2}+d f\right)}\right]$. This parameter permits a direct comparison of the impact of segmentation level on time estimates between the different conditions, irrespective of differences in stimulus type and measurement method. Table 1 indicates that the impact of segmentation level was prominent in the RC condition for both verbal and tactual stimuli, whereas segmentation had no effect on time estimates under the PA condition for either stimulus type. It is also evident that the differential effect of segmentation level cannot be accounted for by memory for the specific stimuli presented, as reflected by recall or recognition. A limitation of the first four experiments is that they do not reveal whether the differential influence of segmentation is attributable to the difference in paradigm, the difference in time estimation method, or to the interaction between these two variables.
Table 1

Summary of the Parameter Values (Means) for Experiments 1-4

\begin{tabular}{|c|c|c|}
\hline \multirow[b]{2}{*}{ Measurement } & \multicolumn{2}{|c|}{ Segmentation Level } \\
\hline & High & Low \\
\hline \multicolumn{3}{|c|}{$\begin{array}{c}\text { Experiment } 1 \\
\text { (RC Condition, Auditory Stimuli) }\end{array}$} \\
\hline $\begin{array}{l}\text { TE* } \\
\text { RNE } \\
\text { FR } \\
\text { CG } \\
\text { HP }\end{array}$ & $\begin{array}{c}218.37 \\
31.21 \\
14.50 \\
21.05 \\
3\end{array}$ & $\begin{array}{c}132.21 \\
27.89 \\
13.25 \\
20.95 \\
3\end{array}$ \\
\hline \multicolumn{3}{|c|}{$\begin{array}{c}\text { Experiment } 2 \\
\text { (PA Condition, Auditory Stimuli) }\end{array}$} \\
\hline $\begin{array}{l}\text { TE } \dagger \\
\text { RNE } \\
\text { FR } \\
\text { CG } \\
\text { HP }\end{array}$ & $\begin{array}{c}94.89 \\
30.73 \\
12.19 \\
20.34 \\
3\end{array}$ & $\begin{array}{c}96.22 \\
32.76 \\
12.69 \\
20.02 \\
3\end{array}$ \\
\hline \multicolumn{3}{|c|}{$\begin{array}{c}\text { Experiment } 3 \\
\text { (RC Condition, Tactual Stimuli) }\end{array}$} \\
\hline $\begin{array}{l}\text { TE* } \\
\text { RNE } \\
\text { FR } \\
\text { HP }\end{array}$ & $\begin{array}{r}218.50 \\
9.50 \\
8.43 \\
2.80\end{array}$ & $\begin{array}{r}140.00 \\
9.06 \\
7.97 \\
2.70\end{array}$ \\
\hline \multicolumn{3}{|c|}{$\begin{array}{c}\text { Experiment } 4 \\
\text { (PA Condition, Tactual Stimuli) }\end{array}$} \\
\hline $\begin{array}{l}\text { TE } \dagger \\
\text { RNE } \\
\text { FR } \\
\text { HP }\end{array}$ & $\begin{array}{r}72.26 \\
9.96 \\
8.00 \\
2.73\end{array}$ & $\begin{array}{r}68.06 \\
9.56 \\
8.53 \\
2.66\end{array}$ \\
\hline
\end{tabular}

Note $-R^{2}$ values (magnitude of experimental effect of segmentation level) for Experiments 1-4 were 0.60, 0.00, 0.45, and 0.00, respectively. $\mathrm{RC}=$ retrospective-comparative; $\mathrm{PA}=$ prospective-absolute; $\mathrm{TE}=$ magnitude of time estimates; RNE = estimated number of stimuli presented during the target interval; FR = number of stimuli correctly recalled; $\mathrm{CG}=$ number of stimuli correctly recognized (corrected for guessing); HP = number of high-priority events correctly recalled. ${ }^{*}$ In millimeters. †In seconds.

\section{EXPERIMENT 5}

In Experiment 5 we used an orthogonal manipulation of paradigm (retrospective vs. prospective) and time estimation method (comparative vs. absolute) in order to reveal whether the influence of segmentation was attributable to differences in paradigm or time estimation method, or to the interaction between them. Furthermore, in order to extend the generality of the findings, we used a shorter clock time and a different ratio of the number of HPEs to non-HPE stimuli than were utilized in the first four experiments. It was predicted that the effect of segmentation level on time estimates would be strongest for the RC condition and that no such effect would be observed for the PA condition. It was further expected that a positive effect would be obtained for the RA and PC conditions, although with a lower magnitude than that for the RC condition.

\section{Method}

Subjects. Eighty subjects participated in the experiment as part of a course requirement. The subjects were randomly assigned in 
equal numbers to eight different conditions resulting from combining the levels of paradigm (prospective vs. retrospective), time estimation method (comparative vs. absolute), and segmentation level (low vs. high).

Stimuli. Two tapes, each including two recordings, were prepared. The first recording contained a 10-sec empty interval, beginning with the word "start" and ending with the word "end." The second recording lasted for $41 \mathrm{sec}$ and included 16 nouns that were selected according to the criteria described in Experiment 1, and three animal names serving as HPEs. In the low-segmentation condition, the HPEs were placed in the first three positions, and in the high-segmentation condition they were placed in Positions 4,9 , and 16. The interstimulus interval was $1 \mathrm{sec}$ and the duration of each word was approximately $1 \mathrm{sec}$. In all other respects the stimuli were identical to those of Experiment 1.

Time estimation method. A major problem with this design was in providing a time estimation method that would enable a direct comparison between comparative and absolute estimates. Thus, the following method was used 1 time estimation form, similar in format to that described in Experiment 1, was designed. When a comparative time estimation was utilized, the procedure was identical to that describe in Experiment 1. However, when time was estimated in an absolute manner, the $30-\mathrm{mm}$ upper line was presented as representing a duration of $10 \mathrm{sec}$, and the subjects were asked to divide the lower line so that its left segment represented the duration of the word list. The empty recording was not mentioned.

Procedure. The procedure was identical both to that described in Experiment 1 for the retrospective conditions and in Experiment 2 for the prospective conditions. The difference between absolute and comparative conditions was in the utilization of the different time estimation methods. The subjects were tested individually. They first completed the time estimation task and then were asked to estimate the RNE. Following that, they were presented with a recognition test consisting of 38 words. This list included 16 new ordinary words, 3 new animal names, and the 16 ordinary words and 3 animal names that had previously appeared in the recording. The sequence of words was randomized and the subjects were asked to mark the words that had appeared in the recording. The recall and recognition tests produced parallel patterns of responses in the previous experiments, so only a recognition test was used. A recognition measure is superior to a recall measure in this context, because the former could be corrected for guessing.

\section{Results and Discussion}

The parameters that were computed for each subject were (1) magnitude of time estimates in millimeters (TE), (2) the RNE measure, (3) number of stimuli correctly recognized after correction for guessing (CG), and (4) the number of HPEs correctly recognized after correction for guessing (HP). The means of the above measures are presented in Table 2 . The magnitude of experimental effects of segmentation level $\left(R^{2}\right)$ was computed in each condition, as described previously. These values are also presented in Table 2, enabling a direct comparison with the Table 1 summary of Experiments 1-4. The data were analyzed by a three-way analysis of variance (paradigm $x$ method $x$ segmentation level). The only significant effect obtained for the memory measures was a main effect of measurement method on HP; HPEs were better recognized in the comparative than in the absolute method $[F(1,72)=25.04, p<.01]$. This replicates the findings of the previous experiments and further supports the notion that segmentation effects are not the direct consequence of remembering the presented events.
Table 2

Means and Standard Deviations of the Parameter Values for Experiment 5

\begin{tabular}{|c|c|c|c|c|}
\hline \multirow[b]{3}{*}{ Measurement } & \multicolumn{4}{|c|}{ Segmentation Level } \\
\hline & \multicolumn{2}{|c|}{ High } & \multicolumn{2}{|c|}{ Low } \\
\hline & $M$ & $S D$ & $M$ & $S D$ \\
\hline \multicolumn{5}{|c|}{ Retrospective-Absolute } \\
\hline TE & 163.80 & 36.00 & 130.70 & 29.93 \\
\hline RNE & 18.30 & 2.36 & 19.00 & 2.00 \\
\hline CG & 13.80 & 2.35 & 14.00 & 3.56 \\
\hline HP & 2.70 & 0.48 & 2.70 & 0.48 \\
\hline \multicolumn{5}{|c|}{ Prospective-Absolute } \\
\hline TE & 136.90 & 37.77 & 133.70 & 36.02 \\
\hline RNE & 15.90 & 3.00 & 17.20 & 1.55 \\
\hline CG & 14.80 & 1.48 & 15.10 & 2.35 \\
\hline HP & 2.50 & 0.53 & 2.50 & 0.53 \\
\hline \multicolumn{5}{|c|}{ Retrospective-Comparative } \\
\hline TE & 210.60 & 31.87 & 112.50 & 35.65 \\
\hline RNE & 20.00 & 2.75 & 19.60 & 2.76 \\
\hline CG & 15.20 & 2.49 & 15.90 & 2.42 \\
\hline HP & 3.00 & 0.00 & 3.00 & 0.00 \\
\hline \multicolumn{5}{|c|}{ Prospective-Comparative } \\
\hline TE & 157.10 & 35.95 & 130.40 & 28.26 \\
\hline RNE & 19.00 & 1.56 & 18.20 & 2.39 \\
\hline $\mathrm{CG}$ & 13.70 & 1.64 & 13.30 & 3.16 \\
\hline HP & 3.00 & 0.00 & 3.00 & 0.00 \\
\hline
\end{tabular}

Note- $\boldsymbol{R}^{2}$ values (magnitude of experimental effect of segmentation level) for the retrospective-absolute, prospective-absolute, retrospective-comparative, and prospective-comparative paradigms were $0.19,0.043$, 0.82 , and 0.38 , respectively. TE (in millimeters) $=$ magnitude of time estimates; RNE = estimated number of stimuli presented during the target interval; $\mathrm{CG}=$ number of stimuli correctly recognized (corrected for guessing); HP = number of high-priority events correctly recognized (corrected for guessing).

An analysis of the time estimation measures indicated that estimates were higher for the retrospective than for prospective conditions $[F(1,72)=4.21, p<.05]$, and higher for high- than for the low-segmentation level $[F(1,72)=30.83, p<.01]$. The main effect of measurement method was not significant $(p=.12)$. The two significant interactions were paradigm $\times$ segmentation level $[F(1,72)=12.19, p<.01]$ and measurement method $\times$ segmentation level $[F(1,72)=9.30, p<.01]$. Further contrast analyses (least squares means) clarified these interactions. In the retrospective conditions, the time estimates were higher when segmentation level was high than when it was low $[t(72)=6.39, p<.01]$, whereas under prospective conditions the respective contrast was not significant $(p=.14)$. When a comparative measurement method was used, the time estimates were higher for the high- than for the low-segmentation level $[t(72)=$ $6.08, p<.01]$, but when an absolute method was used the respective difference was not significant $(p=.08)$.

Contrasts were also computed between time estimates obtained under high- and low-segmentation levels in the RA, RC, PA, and PC conditions. Time estimates were significantly higher for the high-segmentation level than for the low-segmentation level only in the RC $[t(72)=$ $6.76, p<.01]$ and RA $[t(72)=2.28, p<.05]$ condi- 
tions. In the PA and PC conditions, the respective contrasts were not significant $(p=.82$ and $p=.07$, respectively). Multiple regressions with segmentation level RNE and CG as independent variables and TE as the dependent variable were computed for each of the four conditions. In the RC condition, the multiple regression was .79 $[F(3,16)=7.73, p<.01]$, the beta value of segmentation level was .89 , and this was the only variable with a significant contribution. For the RA condition, the multiple regression was $.25[F(3,16)=2.30, p<.05]$ and the beta value of the segmentation level was .5. For the PA condition, the multiple regression was not significant and the beta value of the segmentation level was .02. For the PC condition, the multiple regression was .16 (n.s.) and the beta value of the segmentation level was .38 .

\section{GENERAL DISCUSSION}

The present five experiments demonstrate the differential role of segmentation level in time estimation under different combinations of paradigm and measurement methods. The first experiment, replicating previous findings (Poynter, 1983; Poynter \& Homa, 1983), showed that when duration estimates are measured under RC conditions, the higher the segmentation level of a verbal message presented during the target interval, the longer the perceived duration of that interval. The second experiment failed to reveal the same effect when duration estimates were measured under PA conditions. The results of these two experiments also indicate that this difference cannot be explained by differences in memory for events. This conclusion is derived from the findings that showed no differences in recall or recognition for either ordinary stimuli or HPEs under the various conditions, and no significant effects of these memory parameters in the multiple regression equations. The fact that time estimation is strongly influenced by subjects' awareness of estimation requirements (i.e., paradigm), and by the specific method of measurement, or both, supports the notion that it is a context-dependent process (Block, 1989) that resembles cognitive operations' mediating judgmental tasks (e.g., Payne, 1982). The generality of the present findings was supported in Experiments 3 and 4, which produced similar results for tactual stimuli. Thus, the processes that mediate time estimation are modality independent.

In Experiment 5, the generality of these findings was further extended by using a different clock time and a different ratio of HPEs to non-HPE stimuli. The pattern of results obtained throughout the study is best explained by the notion that PA estimates are mediated by real-time temporal information processing, whereas RC estimates are based on retrieval of contextual-change-related information stored during the estimated interval. Hence, realtime temporal information processing produces an ongoing register of attended temporal units (e.g., Boltz, 1991; Thomas \& Brown, 1974; Thomas \& Weaver, 1975), which provides the basis for time estimates. Consequently, the number of HPEs or contextual changes occurring dur- ing the estimated interval is irrelevant for this type of time estimation process. However, when duration estimates are obtained under RC conditions, ongoing temporal information processing is carried out accidentally and unintentionally, and the temporal information stored in the cognitive timer cannot serve as a reliable source for time estimation. Thus, substitute information, such as the number of HPEs or contextual changes, needs to be retrieved from memory in order to provide a better estimate for the duration of the interval. The amount of retrievable prominent information is related to the number of HPEs or contextual changes encountered during the estimated interval; thus, the higher the number of presented HPEs, the longer the comparative retrospective duration estimate.

In the RA and PC conditions, the time estimates were a function of both sources of information, with the relative contribution of each determined by its perceived representativeness of the estimated duration. Indeed, the magnitude of the effects obtained for segmentation level clearly indicates that its contribution to time estimation was highest under the RC condition, intermediate under RA and PC conditions, and nonexistent under the PA condition. It is plausible that these magnitudes are a reflection of the relative role of memory processes in time estimates under each of these conditions. However, these might only reflect the specific conditions of the present experiment.

The present results further show that when HPEs are dispersed throughout the entire interval, they produce longer perceived durations than when they are grouped together. Hence, the major factor mediating the perception of duration is the changes in meaning rather than the number of HPEs per se. The magnitude of the segmentation effect was higher when the ratio of HPEs to nonHPE stimuli was lower (Experiment 5, RC condition) than when it was higher (Experiments 1 and 2). This might indicate that this ratio is also important in determining the impact of segmentation, but this conclusion must be tested further.

The nature of retrieving information under RC conditions may generally be similar to the process proposed for remembering the time of past events (Friedman, 1993). Thus, when the memory system receives a request for time estimation of a previously presented interval, it may activate a rapid scanning of memory storage during which prominent stored information, such as HPEs and other external or contextual changes, is retrieved. The number of meaningful segments is counted and then transformed into an analog representation of the estimated duration. As hypothesized earlier, this information influences time estimates to a lower extent in the RA and PC conditions and not at all in the PA condition.

It is interesting to note that the higher the segmentation level, the lower the accuracy of time estimates, which suggests that segmentation produces perceptual distortions. However, as in many other perceptual examples, the mechanism responsible for perceptual distortions, in fact, facilitates interaction with the environment under normal 
conditions (e.g., the Müller-Lyer illusion and perceptual constancies). It may well be that in real-life situations, time estimation processes will be disrupted when the perceiver is confronted with many salient HPEs.

In this respect, the present study might lack ecological validity because it presents a high ratio of HPEs to nonHPE stimuli and artificially directs attention to HPEs. These conditions may not fully represent natural environments, as real-life situations provide context and meaning that are absent in laboratory situations. On the other hand, by enhancing the segmentation effect, its role in time estimation processes can be more easily revealed. Future research should address the tradeoff between time estimation performed under controlled laboratory conditions and that performed under conditions of natural environment.

\section{REFERENCES}

BADDEley, A. (1990). Human memory, theory and practice. Boston: Allyn \& Bacon.

BERLYNE, D. E. (1966). Effects of spatial order and inter-item interval on recall of temporal order. Psychonomic Science, 6, 375-376.

BLock, R. A. (1974). Memory and the experience of duration in retrospect. Memory \& Cognition, 2, 153-160.

Block, R. A. (1978). Remembered duration: Effects of event and sequence complexity. Memory \& Cognition, 6, 320-326.

BLOCK, R. A. (1989). Experiencing and remembering time: Affordance, context, cognition. In I. Levin \& D. Zakay (Eds.), Time and human cognition: A life span perspective (pp. 333-364). Amsterdam: NorthHolland.

BLOCK, R. A. (1992). Prospective and retrospective duration judgment: The role of information processing and memory. In F. Macar, V. Pouthas, \& W. J. Friedman (Eds.), Time, action and cognition (pp. 141153). Dordrecht: Kluwer Academic Publishers.

Block, R. A., \& ReED, M. A. (1978). Remembered duration: Evidence for a contextual change hypothesis. Journal of Experimental Psychology: Human Learning \& Memory, 4, 656-665.

Boltz, M. (1991). Time estimation and attentional perspective. Perception \& Psychophysics, 49, 422-433.

Clausen, J. (1950). An evaluation of experimental methods of time judgment. Journal of Experimental Psychology, 40, 756-761.

FRIEDMan, J. W. (1993). Memory for the time of past events. Psychological Bulletin, 113, 44-66.

Grondin, S., \& Macar, F. (1992). Dividing attention between temporal and nontemporal tasks: Preliminary results of a performance characteristic-POC-analysis. In V. Pouthas, F. Macar, \& W. J. Friedman (Eds.), Time, action and cognition (pp. 119-129). Dordrecht: Kluwer Academic Publishers.
Hicks, R. E., Miller, G. W., Gaes, G., \& Bierman, K. (1977). Concurrent processing demands and the experience of time in passing. American Joumal of Psychology, 90, 413-446.

Hicks, R. E., Miller, G. W., \& Kinsbourne, M. (1976). Prospective and retrospective judgments of time as a function of amount of information processes. American Journal of Psychology, 89, 719-730.

JONES, M. R., \& BoLTZ, M. (1989). Dynamic attending and responses to time. Psychological Review, 96, 459-491.

MCCLAIN, L. (1983). Interval estimation: Effect of processing demands on prospective and retrospective reports. Perception \& Psychophysics, 34, $185-189$.

Ornstein, R. E. (1969). On the experience of time. Middlesex, England: Penguin Books.

PAyne, J. W. (1982). Contingent decision behavior. Psychological Bulletin, 92, 382-402.

Poynter, W. D. (1983). Duration judgment and the segmentation of experience. Memory \& Cognition, 11, 77-82.

PoYNTER, W. D. (1989). Judging the duration of time intervals: A process of remembering segments of experience. Inferring time's passage. In I. Levin \& D. Zakay (Eds.), Time and human cognition: $A$ life span perspective (pp. 305-329). Amsterdam: North-Holland.

Poynter, W. D., \& Homa, D. (1983). Duration judgment and the experience of change. Perception \& Psychophysics, 33, 548-560.

RoSENTHAL, R. (1984). Meta-analytic procedure for social research. Beverly Hills: Sage Publications.

Thomas, E. A. C., \& Brown, I., JR. (1974). Time perception and the filled-duration illusion. Perception \& Psychophysics, 16, 449-458.

Thomas, E. A. C., \& Weaver, W. B. (1975). Cognitive processing and time perception. Perception \& Psychophysics, 17, 363-367.

ZAKAY, D. (1989). Subjective time and attentional resource allocation: An integrated model of time estimation. In I. Levin \& D. Zakay (Eds.), Time and human cognition: A life span perspective (pp. 365-395). Amsterdam: North-Holland.

ZAKAY, D. (1990). The evasive art of subjective time measurement: Some methodological dilemmas. In R. A. Block (Ed.), Cognitive models of psychological time (pp. 59-83). Hillsdale, NJ: Erlbaum.

ZAKAY, D. (1992a). On prospective time estimation, temporal relevance and temporal uncertainty. In V. Pouthas, F. Macar, \& W. J. Friedman (Eds.). Time, action and cognition (pp. 109-119). Dordrecht: Kluwer Academic Publishers.

ZAKAY, D. (1992b). The role of attention in children's time perception. Journal of Experimental Child Psychology, 54, 335-371.

ZAKAY, D, \& FALLACH, E. (1984). Immediate and remote time estimation: A comparison. Acta Psychologica, 57, 69-81.

ZaKay, D., \& Feldman, T. (1993). The role of segmentation and of recallability in time estimation. Psychological Record, 43, 415-428.

ZakaY, D., Nitzan, D., Glicksohn, J. (1983). The influence of task difficulty and external tempo on subjective time estimation. Perception \& Psychophysics, 34, 451-456.

(Manuscript received November 16, 1992; revision accepted for publication July 19, 1993.) 